\title{
Thyroid dysfunction in thalassaemic patients: ferritin as a prognostic marker and combined iron chelators as an ideal therapy
}

\section{Valeria Chirico', Lacquaniti Antonio ${ }^{2,3}$, Salpietro Vincenzo' ${ }^{1}$, Nicoletta Luca', Ferraù Valeria', Piraino Basilia', Rigoli Luciana', Salpietro Carmelo' ${ }^{1}$ and Arrigo Teresa'}

${ }^{1}$ Departments of Pediatric Sciences, ${ }^{2}$ Internal Medicine, University of Messina, Messina, Italy and ${ }^{3}$ Department of Internal Medicine, Mediterranean Institute for Transplantation and Advanced Specialized Therapies, ISMETT,

University of Pittsburgh Medical Center, Palermo, Italy
Correspondence

should be addressed to

$\checkmark$ Chirico who is now at

Department of Pediatrics,

98100 Messina, Italy

Email

valeriachirico@hotmail.it

The journal and the authors apologise for errors in the display of names of the authors in the above article published in the European Journal of Endocrinology (vol 169, pp 785-793) and in the Erratum published in the February issue (vol 170 X1 DOI: 10.1530/EJE/13-0627e). The correct names and their affiliations are published in full below:

Valeria Chirico ${ }^{1}$, Antonio Lacquaniti ${ }^{2,3}$ Vincenzo Salpietro $^{1}$, Nicoletta Luca $^{1}$, Valeria Ferraù ${ }^{1}$, Basilia Piraino ${ }^{1}$, Luciana Rigoli $^{1}$, Carmelo Salpietro ${ }^{1}$ and Teresa Arrigo ${ }^{1}$

${ }^{1}$ Departments of Pediatric Sciences, ${ }^{2}$ Internal Medicine, University of Messina, Messina, Italy and ${ }^{3}$ Department of Internal Medicine, Mediterranean Institute for Transplantation and Advanced Specialized Therapies, ISMETT, University of Pittsburgh Medical Center, Palermo, Italy

(Correspondence should be addressed to V Chirico who is now at Department of Pediatrics, 98100 Messina, Italy; Email: valeriachirico@hotmail.it) 